\title{
Penile Cellulitis Due to Methicillin Resistant Staphylococcus aureus MRSA in a 15 Month Old Uncircumcised Boy: A Case Report
}

\author{
Abiezer Disla*, Ester Flores, Carlos Guerra, and Evan Finlay \\ Department of Pediatrics, Family Health Care Network, California, USA
}

*Corresponding author: Abiezer Disla, Department of Pediatrics, Family Health Care Network, California, USA, E-mail: abiezerd20@gmail.com

Received: 01 Aug, 2020 | Accepted: 12 Aug, 2020 | Published: 18 Aug, 2020

Citation: Disla A, Flores E, Guerra C, Finlay E (2020) Penile Cellulitis Due to Methicillin Resistant Staphylococcus aureus MRSA in a 15 Month Old Uncircumcised Boy: A Case Report. J Clin Case Stu 5(3): dx.doi.org/10.16966/2471-4925.205

Copyright: (C) 2020 Disla A, et al. This is an open-access article distributed under the terms of the Creative Commons Attribution License, which permits unrestricted use, distribution, and reproduction in any medium, provided the original author and source are credited.

\section{Abstract}

Penile cellulitis is an uncommon clinical condition. Most of the cases are due to infection by Group B Streptococcus (GBS) and presents in young adults. In the pediatric age, it is a complication of balanitis or balanoposthitis and most of cases respond to appropriate antibiotics with resolution of local and systemic symptoms which avoids complications Administration of appropriate antibiotics resolves local and systemic symptoms and avoids complications. To date, there are no cases reported in literature of penile cellulitis caused by Methicillin Resistant S. aureus (MRSA) in children.

Here, we present a case of a 15-month-old uncircumcised boy with penile cellulitis due to MRSA. The clinical presentation, etiology and management are discussed.

Keywords: Penile cellulitis; S. aureus; Balanoposthitis; Streptococcus

Abbreviations: GBS: Group B Streptococcus; MRSA: Methicillin resistant S. aureus; TMP-SMX: Trimethoprim/Sulfamethoxazole; CA-MRSA: Community Acquired Methicillin Resistant Staphylococcus aureus

\section{Case Presentation}

A 15-month-old uncircumcised male with an unremarkable past medical history was admitted to the pediatric unit due to worsening swelling of the penis and difficulty urinating. Initially, patient had fussiness and edema of the prepuce with purulent discharge. He was first evaluated at Urgent Care, where he was prescribed oral Cephalexin (Keflex), oral Trimethoprim/Sulfamethoxazole (Bactrim), and topical Mupirocin. Patient's swelling increased and he then developed difficulty voiding. He was subsequently taken to the Emergency Department where cultures were obtained and he was prescribed a topical antifungal cream, Clotrimazole, and sent home. Patient was seen the following day at his pediatrician's office for follow-up. He did not present with fever or any other systemic signs of infection. On his physical exam there was significant swelling with erythema, purulent discharge, and extreme tenderness of the entire penis. His prepuce was unable to be retracted, hence his glans and meatus were not visualized (Figure 1). He did not have any abdominal pain or lymphadenopathies. He was then directly admitted to the pediatric unit for intravenous Clindamycin. Cultures were obtained from the purulent secretions; Methicillin resistant $S$. aureus was isolated and found to be sensitive to Clindamycin; resistant to Trimethoprim/ Sulfamethoxazole and Oxacillin.
Within 24 hours the swelling and redness of the penis showed evidence of improvement. He was able to void without difficulty and was discharged home after 48 hours of intravenous antibiotics. He completed a 7-day outpatient course of oral Clindamycin. Upon follow-up, patient had recovered without any complications.

\section{Discussion}

Penile cellulitis presents as swelling of the penis and may be associated with discharge as well as inguinal lymphadenopathy. It may also produce urinary symptoms as well as systemic toxicity. Although uncommon, is predominantly seen in sexually active young men. It can, however, affect all age groups including pediatric population such as newborns and young children [1].

Physiology phimosis is a common finding in uncircumcised newborn males. Due to this, the foreskin is unable to be retracted and adhesions are visualized. If the prepuce is forcefully retracted, it may cause micro traumas which are a predisposing factor causing balanoposthitis. If balanoposthitis not treated adequately, it can lead to cellulitis of the penis.

Given penile cellulitis is usually transmitted sexually, Streptococci are the most common causative organisms isolated. B-hemolytic Group B Streptococci (GBS) is the likely pathogen as it can be 


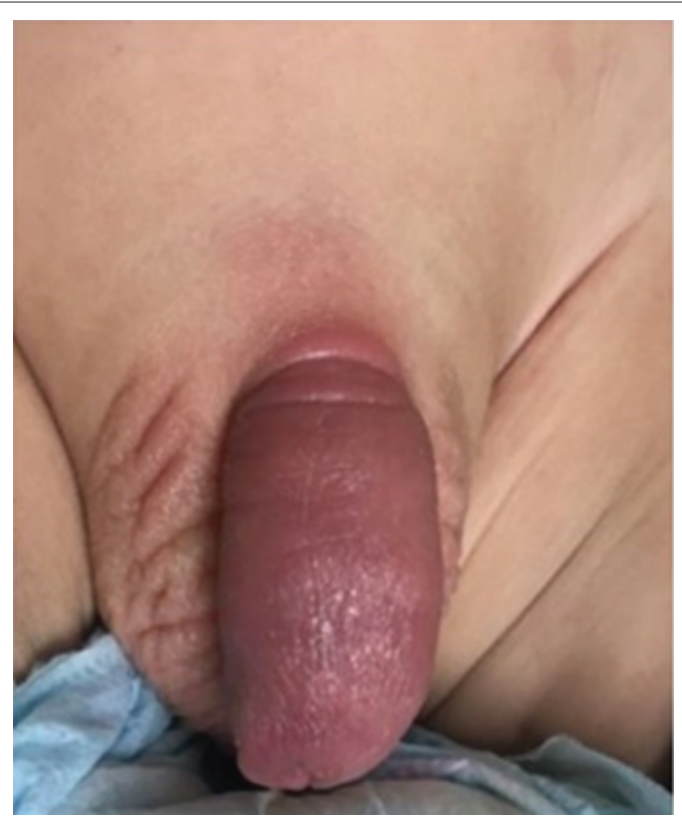

Figure 1: Penile swelling and erythema. Unable to visualize glans of the penis.

transmitted from the female oral cavity or vagina. If purulent discharge is present a sample should be sent to the laboratory for gram staining and culture [1].

In pediatric populations, however, GBS has been reported only in young infants and is exceedingly uncommon in toddlers [2]. Infections caused by Community Acquired Methicillin Resistant Staphylococcus aureus (CA-MRSA) are an emerging problem and is increasing in prevalence among staphylococcal infections [3]. Currently, it has been reported in young pediatrics population, more common in children in day care centers [4].

A recent antibiogram study has reported a new increase in Trimethoprim/Sulfamethoxazole (TMP-SMX) resistance in
Methicillin Resistant Staphylococcus aureus (MRSA) isolate [4]. In the absence of treatment, penile cellulitis may progress rapidly. It should be promptly identified and properly treated to prevent rare severe complications such as gangrene [5]. After proper antibiotics have commenced, there is usually a rapid response and improvement of the symptoms [1]

This case highlights the importance of obtaining cultures from secretions and genital lesions to guide treatment and the need to choose empiric antibiotics based on local resistance patterns [3].

\section{Conclusion}

Prompt evaluation and identification of penile lesions in uncircumcised males should be priority in any male genital exam. Proper management includes evaluation of secretions, culture of the secretions, and initiation of empiric antibiotics. Treatment should be based on local antibiograms. Therapy should be administered rapidly to avoid potential complications. If clinical response is delayed, different coverage should be considered.

Parents of an uncircumcised male should be routinely educated to avoid forceful retractions of the prepuce to prevent microabrasions and penile skin infections.

\section{References}

1. Bardapure M, Vaswani N (2009) Cellulitis of the Penis: A Case Report. BMJ Case Rep 2009: bcr0120091457.

2. Brady MT (1987) Cellulitis of the Penis and Scrotum Due to Group B Streptococcus. J Urol 137: 736-737.

3. Sturgiss EA, Bowden FJ (2008) Penile Cellulitis Due to CommunityAcquired Methicillin-Resistant Staphylococcus aureus in an HIVPositive Man. Int J STD AIDS 19: 423-424.

4. Grundmann H, Aires-de-Sousa M, Boyce J, Tiemersma E (2006) Emergence and Resurgence of Methicillin-Resistant Staphylococcus aureus as a Public-Health Threat. Lancet 368: 874-885.

5. Haury B, Rodeheaver G, Stevenson T, Bacchetta C, Edgerton MT, et al. (1975) Streptococcal Cellulitis of the Scrotum and Penis with Secondary Skin Gangrene. Surg Gynecol Obstet 141: 35-39. 